\title{
Augmenting Physical Buttons with Vibrotactile Feedback for Programmable Feels
}

\author{
Chaeyong Park Jinhyuk Yoon Seungjae Oh Seungmoon Choi \\ Interaction Laboratory, Pohang University of Science and Technology (POSTECH), Republic of Korea \\ $\{$ pcy8201, jinhyukyoon, oreo329, choism\}@ postech.ac.kr
}

\begin{abstract}
Physical buttons provide clear haptic feedback when pressed and released, but their responses are unvarying. Physical buttons can be powered by force actuators to produce unlimited click sensations, but the cost is substantial. An alternative can be augmenting physical buttons with simple and inexpensive vibration actuators. When pushed, an augmented button generates a vibration overlayed on the button's original kinesthetic response, under the general framework of haptic augmented reality. We explore the design space of augmented buttons while changing vibration frequency, amplitude, duration, and envelope. We then visualize the perceptual structure of augmented buttons by estimating a perceptual space for 7 physical buttons and 40 augmented buttons. Their sensations are also assessed against adjectives, and results are mapped into the perceptual space to identify meaningful perceptual dimensions. Our results contribute to understanding the benefits and limitations of programmable vibration-augmented physical buttons with emphasis on their feels.
\end{abstract}

\section{Author Keywords}

Button; Vibrotactile; Haptics; Augmented reality; Multimodal

\section{CCS Concepts}

-Human-centered computing $\rightarrow$ Human computer interaction (HCI); Haptic devices; User studies;

\section{INTRODUCTION}

Physical buttons are one of the most effective, efficient, and pervasive user interfaces widely used for computer keyboards, gamepads, electronic devices, and automobiles, just to name a few. Their simple operation principle and evident physical affordance, as well as natural and pronounced haptic confirmation feedback, are valuable merits. In comparison, virtual buttons, typically displayed on touchscreens, offer a critical advantage of adaptability, but they lack physical affordance and natural haptic feedback. While virtual buttons become more advanced and attractive, physical buttons are also likely to survive and evolve to a newer form.

Permission to make digital or hard copies of part or all of this work for personal or classroom use is granted without fee provided that copies are not made or distributed for profit or commercial advantage and that copies bear this notice and the full citation on the first page. Copyrights for third-party components of this work must be honored. For all other uses, contact the owner/author(s).

UIST '20, October 20-23, 2020, Virtual Event, USA

(C) 2020 Copyright is held by the author/owner(s).

ACM ISBN 978-1-4503-7514-6/20/10.

http://dx.doi.org/10.1145/3379337.3415837

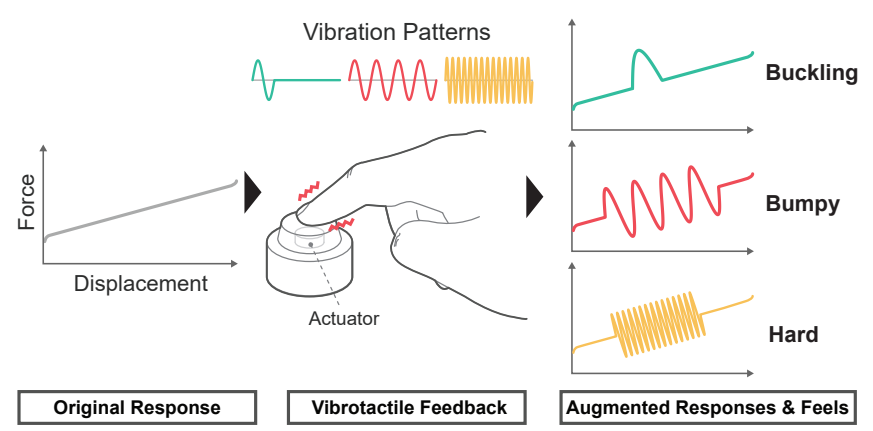

Figure 1. The force response of a physical button is augmented with a vibration generated by an actuator. This simple technology affords a variety of distinct and programmable feels to one physical button.

A physical button always presents the same click sensation. This limits its versatility as a programmable, informative, or affective interface. A natural idea to improve this drawback is adding a force actuator, e.g., a linear motor, to a physical button and modulating its kinesthetic response $[34,35,56]$. When implemented appropriately, this approach provides physically faithful sensations and pleasant experiences. However, a system for such force feedback is generally complex and bulky, thereby incurring a substantial increase in cost.

An alternative approach can be augmenting the feel of a physical button by adding a vibration (Figure 1). This idea was briefly presented earlier in $[39,53]$. When the user presses the button, a vibration signal is generated by the actuator and presented to the user together with the original force response of the button. This approach is based on haptic augmented reality (AR), where real (force) and artificial (vibration) haptic stimuli are combined for mixed sensations [23]. It is also one case of multimodal haptic rendering, where different kinds of haptic (force and vibration) signals are seamlessly blended [62].

In this paper, we explore the vast design space of vibrationaugmented physical buttons in order to acquire fundamental and essential knowledge on their perceptual characteristics. To a physical button of a linear force-displacement response, 40 vibrations of different frequencies, amplitudes, durations, and envelopes are paired. Using these 40 augmented buttons and another 7 physical buttons, we performed two perceptual user experiments. Experiment 1 aimed to visualize the perceptual structure of the augmented and physical buttons by estimating their perceptual space ${ }^{1}$. In Experiment 2, the 47 physical and augmented buttons were rated against seven bipolar adjective 
pairs and three unipolar semantic words. The rating results were projected into the perceptual space to find meaningful perceptual dimensions. These procedures allow us to represent the percepts of the augmented and physical buttons in a 2D Euclidean space and recommend a few pairs of the perceptual axes that span the perceptual space appropriately. From these results, we draw guidelines for designing vibration-augmented physical buttons with desired feels.

The contributions of this work lie on the structured and detailed analysis results on the perceptual attributes of the button-click sensations of vibration-augmented physical buttons using perceptual space and perceptual dimensions.

\section{RELATED WORK}

\section{Haptic Augmented Reality}

Haptic AR is a concept extended from the classical definition of visual AR, where a user experiences real haptic stimuli to which artificial haptic signals are added [23]. Haptic AR enables modulating the material properties of real objects, such as stiffness [20,21] and friction [24], and creating a virtual haptic object within a real object, e.g., a virtual tumor in a real body [22], by means of force feedback.

Tactile feedback can also augment real haptic experiences. For instance, an elastic pad explored by a rigid stick with additional vibration can mimic other materials, such as rubber, wood, and aluminum [13]. Transmitting a vibration to a stylus scanning a touchscreen can render a virtual texture [50]. Touching a real rigid object using a vibration-emitting stylus changes the perceived roughness and friction of the object [9]. Applying an electrical stimulus to the scanning finger can alter the roughness of the real material [64]. Providing electrovibration feedback can augment real surface textures [3].

Haptic AR can reshape the haptic sensations of real objects while preserving the benefits of AR: simpler and cheaper than pure virtual reality (VR). This advantage has inspired us to apply vibrotactile augmentation to physical buttons.

\section{Multimodal Haptic Rendering}

Multimodal haptic rendering is an emerging topic where different types of haptic stimuli are combined to provide more realistic (or otherwise improved) haptic sensations. Highly realistic haptic simulation using a single actuator is impossible at present, and the multimodal approach is an endeavor to overcome the problem. Many advanced (yet complicated) haptic devices that generate multiple stimuli of force, vibration, impact, or heat to a single or multiple body spots have been developed; see [62] for review.

To our research context, rendering methods that blend force, vibration, and impact are particularly relevant. Such examples have been reported for VR to improve the simulation realism of touching stiff surfaces [31, 42] and textures [54]. An ungrounded stylus that alters the roughness and friction of a real surface by presenting textural vibration and braking force

\footnotetext{
${ }^{1}$ Victor et al. [60] explain that "a perceptual space is a mental workspace of points in a sensory domain that supports similarity and difference judgments and enables further processing such as classification and naming."
}

is a unique multimodal example for AR [9]. In our case, a real kinesthetic response that includes both (fast) impulse and (slow) pressing components are augmented by a vibration.

\section{Perceptual Space and Dimensions}

A perceptual space is an $n$-dimensional Euclidian space that visualizes the intrinsic metric relationships between stimuli based on perceptual similarity [65]. A perceptual space is constructed from the perceptual distances estimated between the stimuli of interest. It is often accompanied by a rating procedure in which the stimuli are subjectively evaluated against a set of adjectives or semantic words. Results are mapped into the perceptual space to obtain extrinsic geometric interpretations of the stimuli, namely, perceptual dimensions.

This approach has been an exceedingly effective tool in comprehending the perceptual structure of many types of haptic stimulus, e.g., texture [16, 38, 40], vibration [17, 18, 25, 44, 47, 57], and material [58]. In particular, the sensations of button press were analyzed using perceptual spaces for 10 real and 14 force-feedback buttons [32], 23 physical buttons [36], and 18 touchscreen virtual buttons [10]. We report a perceptual space for physical and vibration-augmented buttons and their extrinsic perceptual properties using a set of adjectives.

\section{Characteristics of Physical Buttons}

Physical buttons are universal in input interfaces. During operation, they convey distinct haptic sensations through both kinesthetic and tactile stimuli. Designing a physical button that has the "right" feel for its purpose and environment has been a classical challenge in physical interface design. For example, Weir et al. analyzed the physical responses of three switches using force, position, and velocity profiles [63]. Alexander et al. characterized the physical properties of 1515 push buttons under multiple categories of size, shape, trigger, force, travel distance, etc. [1].

Despite such efforts, designing and manufacturing physical buttons for desired functions and feels is still a very costly process. Also, the perceptual and cognitive attributes of physical buttons cannot be changed. We try to alleviate this drawback by providing programmable vibrotactile feedback.

\section{Haptic Feedback for Virtual and Physical Buttons}

Research has seen considerable efforts to afford compelling haptic feedback to virtual buttons displayed on touchscreens, for almost 20 years [11]. Conceptually, it corresponds to haptic AR since the real tactile stimulus of touching the surface is altered with an actuator-generated vibration. Park et al. designed 72 tactile signals using linear resonant actuators for virtual button clicks and evaluated them as to the similarity to real-button click sensations [46]. Chen et al. followed an information-theoretic approach to quantify the number of completely identifiable key-click signals produced by piezoelectric actuators [7]. Tactile feedback indeed enhances the typing performance on a virtual keyboard [5, 15, 27]. Furthermore, recent premium mobile phones present very realistic tactile key-click feedback (incredibly short trigger time and duration) using advanced actuators and control technologies [2]. 


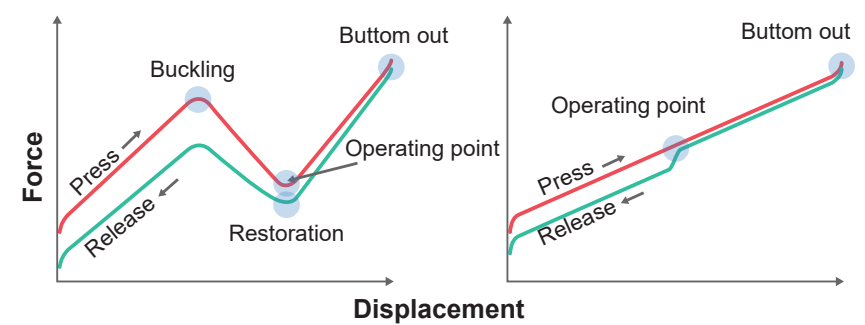

Figure 2. Force-displacement profiles of physical buttons. (Left) General profiles exhibiting buckling and restoration. (Right) Linear profiles without apparent pulses of force.

The above methods substitute the kinesthetic feedback from real key-clicks with vibrotactile feedback. As such, it is difficult to imitate the translational sensation of a physical button. Kim et al. attempted to overcome this limitation by issuing different vibrations in each state of the button press and release $[29,30]$. Sadia et al. rendered data-driven digital buttons on touchscreens by analyzing the force, acceleration, and voltage data from physical buttons [51]. Vibrations were designed to utilize the haptic illusion of compliance [26] so that they would deliver a sense of displacement.

We found two previous instances that augmented physical buttons with vibrotactile feedback. To our knowledge, Ogawa et al. were the first in presenting the idea and built a compact prototype [39]. Shim and Lee applied this approach to buttons in a gamepad and demonstrated the hardware [53]. However, none of them reported physical or perceptual evaluation results on the augmentation. Our work expands the idea by designing a large number of augmented buttons and formally quantifying the perceptual effects of vibrotactile augmentation.

\section{AUGMENTING PHYSICAL BUTTONS}

\section{Hardware}

The perceptual features of a physical button can be represented by its force-displacement profile [29]. When the button is pressed, the response force initially increases as the displacement increases, and then it decreases suddenly from a certain displacement (called buckling [56]; Figure 2, left). When the key reaches the bottom plate, the displacement is saturated, and the response force increases abruptly. When released, the above process is reversed, involving a point for rapid restoration. A button not exhibiting apparent buckling is called a linear switch (Figure 2, right). Several mechanisms are used in switches to provide restoration, such as a metal sheet, a piece of rubber, and a spring. Their mechanical designs and manufacturing systems are fine-tuned to control the delicate feels of key press.

The overall design of our augmented button hardware is shown in Figure 3. We use a linear mechanical switch (Cherry MX Black; maximum displacement $4 \mathrm{~mm}$ ). Since its response does not include salient pulses, vibrotactile augmentation effects can be maximized. A round cap is put on the switch, and the switch is placed into a housing. Both the cap and housing were 3D printed using polylactide filament.

The housing also contains a voice coil type vibration actuator (Haptuator BM3C, Tactile Labs) clenched by bolt binding. The

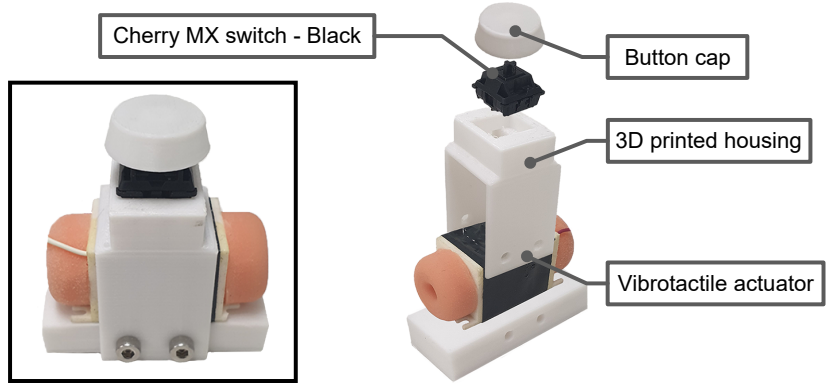

Figure 3. Augmented physical button. It is $51 \mathrm{~mm} \times 25 \mathrm{~mm} \times 55 \mathrm{~mm}$ in size and $55 \mathrm{~g}$ in weight. The button cap has $17.5 \mathrm{~mm}$ of contact diameter and $6.6 \mathrm{~mm}$ of height.

actuator has a wide frequency bandwidth from 30 to $1000 \mathrm{~Hz}$ and a large maximum vibration amplitude up to $10 \mathrm{G}$. This advantage allows us to explore a much greater design space for vibrations, compared to using other smaller piezoelectric actuators or vibration motors. The switch and actuator are connected to Arduino Due for contact detection and vibration waveform generation, which communicates with a PC.

\section{Vibration Profiles}

We designed 40 vibration waveforms by varying three variables: envelope, frequency, and amplitude. The vibration command is computed by

$$
x(t)=A E(t) \sin (2 \pi F t),
$$

where $x(t)$ is the vibration signal at time $t, A$ is the amplitude, $E(t)$ is the envelope function, and $F$ is the frequency.

For frequency, we selected four values: 40, 100, 250, and $400 \mathrm{~Hz}$. For mechanoreceptors, they cover a wide spectrum ranging from a very low frequency mediated by the RA (rapidly adapting) 1 channel to a very high frequency processed by the PC (Pacinian) channel [4]. The lowest-frequency vibration feels like fluttering, while the two highest frequency stimuli $(250$ and $400 \mathrm{~Hz}$ ) are perceived as smooth vibrations.

We designed five envelope functions emulating the key pressrelease operation. In general, key compression takes $20-30 \mathrm{~ms}$, and the entire operation is finished in less than $100 \mathrm{~ms}$ [49]. Three envelopes are rectangular with different durations (25, 50 and $100 \mathrm{~ms}$ ). The other two have the same duration of $100 \mathrm{~ms}$ but decay exponentially with different rates, such that

$$
E(t)=e^{-\tau t},
$$

where $\tau$ is the decay rate (16 and 39). Such exponentiallydecaying sinusoidal waveforms are widely used to indicate virtual collisions [41, 42, 45].

Combining the four frequencies and five envelopes gives 20 waveforms (see Figure 4). When integrated, the 100-ms rectangular envelope (R100) has an area twice of the 50-ms rectangular envelope (R50). The same can be said to R50 and R25 (the 25-ms rectangular envelope). Likewise, the slow-decaying envelope (DS100) has an area twice of the fast-decaying envelope (DF100). Further, R50 and DS100 have the same area, and R25 and DF100 have the same area. Therefore, for the 


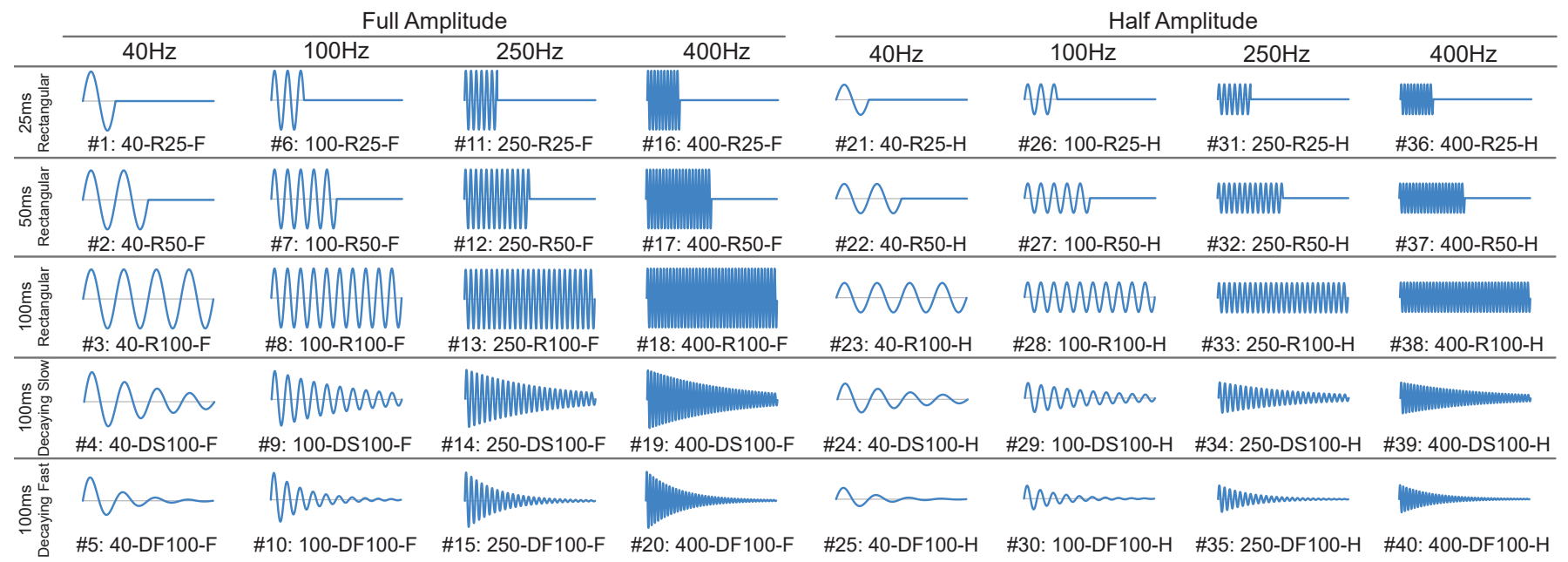

Figure 4. Forty vibration waveforms for button augmentation. Each waveform is represented by a number and a code of parameter values: Frequency $(40,100,250,400)$-Envelope (R25, R50, R100, DS100, DF100)-Amplitude (F, H). Each notation will be used depending on adequacy.

signal energy $P$ represented by the area of the envelope (L1 norm), we have

$$
\begin{aligned}
P(\mathrm{R} 100) & =2 P(\mathrm{R} 50)=4 P(\mathrm{R} 25) \\
& =2 P(\mathrm{DS} 100)=4 P(\mathrm{DF} 100) .
\end{aligned}
$$

We use two levels of amplitude: full and half. The perceptual strength of a vibration also depends on its frequency [19, 33, 59]. Thus, we performed a perceptual experiment for intensity equalization. Given a reference vibration of frequency $400 \mathrm{~Hz}$, five participants found the vibration amplitude that resulted in the same perceptual strength for the other three frequencies. The procedure followed the method of adjustment [12]. It was repeated for both amplitude levels. As a result, the amplitudes for the $40 \mathrm{~Hz}$ stimuli were 0.8 and $0.45 \mathrm{G}$ for the full and half amplitude levels, 1.2 and $0.6 \mathrm{G}$ for $100 \mathrm{~Hz}, 2.3$ and $1.2 \mathrm{G}$ for $250 \mathrm{~Hz}$, and 5.2 and $1.4 \mathrm{G}$ for $400 \mathrm{~Hz}$. This completes the design of the 40 vibrations.

The last important issue is when to initiate vibration playback while the button is pressed [14, 28]. We wait until the linear physical switch is recognized to be pressed. This occurs when the button displacement reaches $2 \mathrm{~mm}$ (out of 4 $\mathrm{mm}$ ). It is sensed by the Arduino controller, and a vibration is immediately produced. This method requires no external sensors. An alternative is to add a displacement sensor and control the vibration initiation timing more precisely on the force-displacement profile. We tested this method and did observe good possibility that the vibration start time would affect the augmented click sensation. However, including this variable as well makes too many stimuli for an experiment that estimates a perceptual space. We focus on the three more elementary design variables of vibration frequency, envelope, and amplitude in the present study and keep the vibration initiation time for future work.

\section{Augmentation Examples}

Figure 5 shows three acceleration and force profiles collected from the augmented buttons. They were measured by an
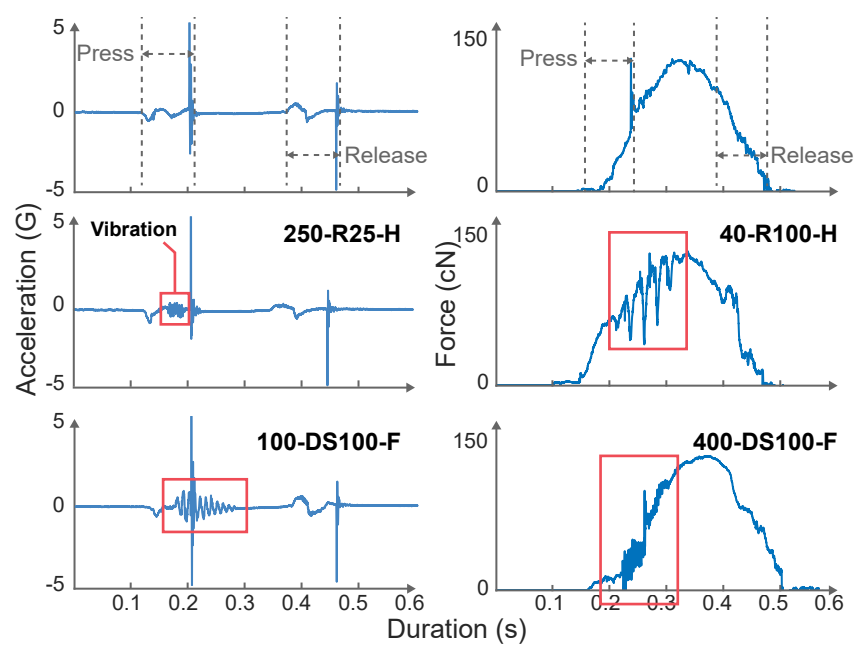

Figure 5. Acceleration and force profiles visualizing the effects of vibration augmentation on physical button click. The top row shows the responses of the physical button itself, and the rest is for augmented ones.

accelerometer (8794A500, Kistler) and a force sensor (FlexiForce, Tekscan). The profiles in the top row are from the physical button itself without vibration feedback. The data meet expectation for key press and release, and also show strong and very narrow peaks at complete compression and restoration. The two panels below show vibration-augmented examples. Each panel demonstrates that a vibration of the commanded frequency, envelope, and amplitude is superimposed immediately after the press is detected. These plots visualize the effect of our vibrotactile augmentation technique on physical button click.

\section{USER EXPERIMENT 1: PERCEPTUAL SPACE}

This experiment aimed to estimate a perceptual space that visualizes the metric relationships between many physical and augmented buttons. Participants' task was to judge the similarity of button-click sensations between the buttons. This perceptual similarity data was converted to perceptual dis- 


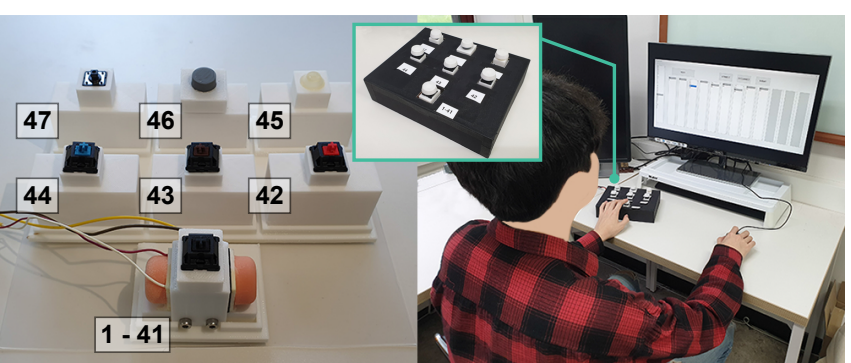

Figure 6. Experimental setup. (Left) One augmented physical button (labeled by "1-41") and six other physical buttons (“42"-“47"). Caps are removed to show the different switches in the buttons. (Right) A participant conducting Experiment 1. The inset shows a box that houses the seven buttons with the identical caps, as well as their labels.

tances, from which a perceptual space was estimated using multidimensional scaling (MDS). This experiment (also Experiment 2) was approved by the Institutional Review Board at the authors' institution (PIRB-2019-E018).

\section{Methods}

\section{Participants}

Twenty participants (10 males and 10 females; mean age 21.2 years) took part in this experiment. No participants reported known sensorimotor abnormalities. They were informed of the experimental conditions and procedure via a written document, and then they signed a consent form.

\section{Apparatus}

In addition to the augmented physical button, we included six other physical buttons of different force-displacement responses for comparison, as shown in Figure 6. To provide identical key-click conditions, we inserted each button into the same 3D-printed housing and put the same cap of diameter $17.5 \mathrm{~mm}$. The button housings were placed on a thin silicon pad $(300 \mathrm{~mm} \times 300 \mathrm{~mm} \times 1 \mathrm{~mm})$ to block vibration propagation to the floor [43] and reduce the lateral movements of the buttons. All the button housings were contained in a large cardboard housing $(300 \mathrm{~mm} \times 300 \mathrm{~mm} \times 45 \mathrm{~mm})$ and were visually hidden from participants. Participants could see only the identical key caps and their labels.

\section{Experimental Conditions}

Table 1 lists the 40 augmented buttons (\#1-\#40, as shown in Figure 4) and the 7 physical buttons (\#41-\#47) without external vibrotactile feedback. The latter selection covers a variety of real physical buttons with different click sensations. Note that button \#41 is the augmented button itself (Cherry MX - Black) without vibration playback.

All of the physical switches have different operating structures. The four Cherry MX switches have different click pressures, stiffnesses, and tactile effects; see [8] for detailed datasheets. For example, button \#42 is also a linear button but requires a smaller force $(0.45 \mathrm{~N})$ to operate than button \#41 $(0.60 \mathrm{~N})$. Both button \#43 and \#44 deliver clear tactile sensations at buckling, but button \#44 makes a louder sound. The membrane switch 2 taken from a remote controller is much stiffer than the membrane switch 1 taken from a keyboard. The tact switch has a unique and very strong click feel, as well as the greatest

\begin{tabular}{cl}
\hline ID \# & Description \\
\hline $1-40$ & Vibration-augmented buttons \\
41 & Cherry MX switch - Black \\
42 & Cherry MX switch - Red \\
43 & Cherry MX switch - Brown \\
44 & Cherry MX switch - Blue \\
45 & Membrane switch 1 - Keyboard \\
46 & Membrane switch 2 - Remote controller \\
47 & Tact switch \\
\hline
\end{tabular}

Table 1. Buttons used in the experiments. The first 40 are vibrationaugmented buttons, while the rest 7 are physical buttons.

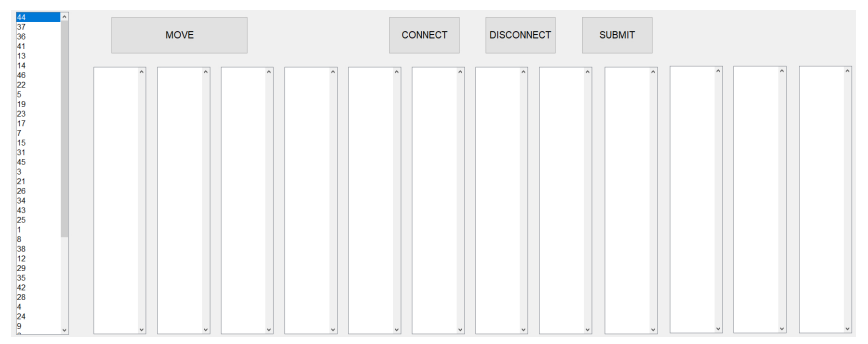

Figure 7. GUI for cluster sorting adapted from [36]. To move a button into a cluster bin, a user selects the button number in the leftmost text box, clicks the 'move' button on the menu, and then chooses the bin.

stiffness. Each physical button conveys a distinct key click sensation and should have a unique force-displacement profile. The 47 conditions were designed to elicit a wide range of tactile sensations for button presses.

\section{Procedure}

For similarity judgment between button click sensations, this experiment used the cluster sorting method [47]. An alternative, direct pairwise similarity rating as in [17], was infeasible because of the large number of stimuli.

Participants sat in a chair in front of a monitor (Figure 6). They pressed the buttons with the left hand and manipulated the experimental program using the mouse held in the right hand. They wore noise-canceling headphones that played white noise to block any sound. Participants' task was to compare the feels of the 47 physical and augmented buttons and group them into a given number of clusters based on their similarity. Each cluster was required to include at least one button. They used a graphical user interface (GUI) shown in Figure 7. Participants were instructed that to feel buttons \#1-\#41, they should select the corresponding number in the leftmost textbox of the GUI and press the (augmented) button at the bottom of the button housing. The numbers for the other physical buttons were marked on the housing.

The experiment had four sessions that respectively used 3, 6, 9 , and 12 clusters. The numbers of clusters were randomly ordered to the four sessions. In addition, to prevent memory effects, stimulus numbers were randomly assigned to the augmented buttons in each session. The physical buttons had the same numbers throughout.

The experiment took one and a half hours on average to complete. Participants rested for a few minutes to prevent fatigue and tactile adaptation between sessions. At the end of the experiment, participants wrote down their clustering criteria. 


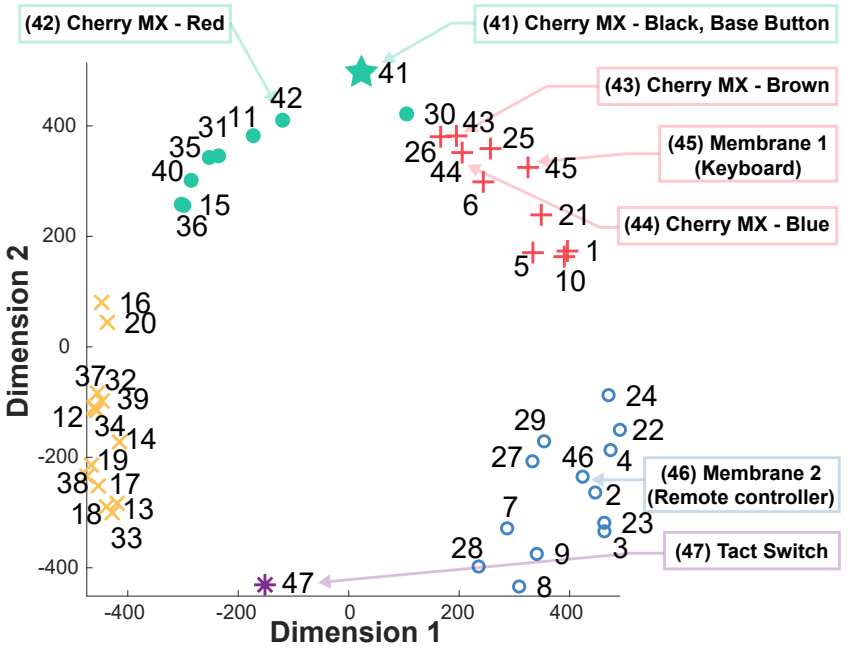

Figure 8. 2D perceptual space of the 47 augmented and physical buttons. The star represents the base physical button \#41 without vibration feedback. Groups found by hierarchical clustering are represented by the same colors and symbols.

\section{Data Analysis}

The data from cluster sorting was processed following the procedure in [47]. The similarity score $s_{i, j}$ between button $i$ and $j$ was initialized to 0 for each participant. If button $i$ and $j$ were grouped in a session with $N$ clusters, $s_{i, j}$ was increased by $N$. This was repeated for all pairs of the buttons. From these similarity scores, a normalized dissimilarity matrix $\left\{d_{i, j} \mid 1 \leq i, j \leq 47\right\}$ was obtained by a linear transformation

$$
d_{i, j}=1000\left(1-\frac{s_{i, j}}{3+6+9+12}\right),
$$

where each pairwise dissimilarity score $d_{i, j}$ is normalized between 0 and 1000. Average scores were calculated from the individual scores. Then, non-metric classical MDS was applied to the dissimilarity matrix to find perceptual spaces with appropriate dimensions. The goodness of fit was evaluated using S-Stress (SS) [52]. SS varies between 0 and 1, and a SS value closer to 0 indicates a better fit.

\section{Results}

To find optional solutions, we examined SS values resulted from MDS while increasing the space dimension. Both 2D and $3 \mathrm{D}$ spaces were adequate $(\mathrm{SS}=0.167$ and $0.114 ; 0.15$ is a recommended cut-off [61]). We thoroughly examined the two perceptual spaces and found that the major results observable from the two spaces were almost the same. For simplicity, we show only the 2D perceptual space in Figure 8, in which the 47 augmented and physical buttons are represented by points.

The overall point distribution in the perceptual space demonstrates that the vibrotactile augmentation of a physical button is a very effective technique in diversifying button-click sensations. This is confirmed by the wide spread of the augmented buttons, almost around a circle. The augmentation effect of some vibrations, e.g., \#8, is even greater (the dissimilarity score $d_{8,41}=970$ where $\# 41$ is the base physical button) than the greatest perceptual difference between the physical buttons $\left(d_{41,47}=935\right)$.

\section{Discussion}

Groups of Physical and Augmented Buttons

For an objective grouping of the buttons, we applied hierarchical clustering using the farthest neighbor metric to the original dissimilarity data. Results are indicated in Figure 8, where the buttons represented by the same symbols and colors belong to the same group. A dendrogram is also provided in the supplemental material (cophenetic correlation 0.8560).

The tact switch \#47 with the greatest stiffness and a strong buckling response forms its own group. The other four groups consist of many augmented or physical buttons.

The green group is anchored by the base physical button for augmentation (\#41, Cherry MX switch - Black). It has another physical button (\#42, Cherry MX switch - Red), which is also a linear button without a buckling sensation [8]. This group has seven augmented buttons (\#11, \#15, \#30, \#31, \#35, \#36, and \#40), and they are characterized by high frequency (250 or $400 \mathrm{~Hz}$ ) and low-energy envelope (R25 or DF); see Figure 4. Button \#30 is the only exception with a low frequency $(100 \mathrm{~Hz})$. Buttons \#16 and \#20, which are also of high frequency and low-energy envelope, are rather the members of the yellow group, but they are not far from the green group. The augmented buttons in this group are closer to the two linear physical buttons \#41 and \#42 than the other physical buttons. This indicates that the button-augmentation effect of vibrations with high frequency and low-energy envelope is relatively weak.

The red group is composed of three physical buttons (\#43, \#44, and \#45; Cherry MX switch - Brown and Blue, and the membrane keyboard switch) and seven augmented buttons (\#1, $\# 5$, \#6, \#10, \#21, \#25, and \#26). The augmented buttons all have low frequency (40 or $100 \mathrm{~Hz}$ ) and low-energy envelope (R25 or DF). Button \#30 of these features is clustered to the green group, but it is also very close to the red group. The two Cherry MX switches, called tactile switches by the manufacturer, make clear buckling sensations. In the perceptual space, button \#43 (Cherry MX switch - Brown) is very close to \#25 and \#26, and \#44 (Cherry MX switch - Blue) is to \#6, \#25, and \#26. Therefore, adding a low-frequency vibration of a lowenergy envelope to a linear button seems to create a similar sensation to mild mechanical buckling. Also, the membrane switch \#45 from a keyboard, which conveys a relatively soft rubber-like elastic feel, is surrounded by augmented buttons of low-frequency and low-energy envelope. This might be related to the compliance illusion elicited by vibration [26].

The average augmentation effects for the green and red groups, quantified by the distance from the base physical button \#41, are weaker than those for the blue and yellow groups.

In the blue group, button \#46 (membrane switch 2), which gives a highly-stiff rubbery feel, is the only physical button. This group has 12 augmented buttons: \#2, \#3, \#4, \#7, \#8, \#9, $\# 22$, \#23, \#24, \#27, \#28, and \#29. They all have low frequency $(40$ or $100 \mathrm{~Hz})$ and medium- or high-energy envelope (DS, 


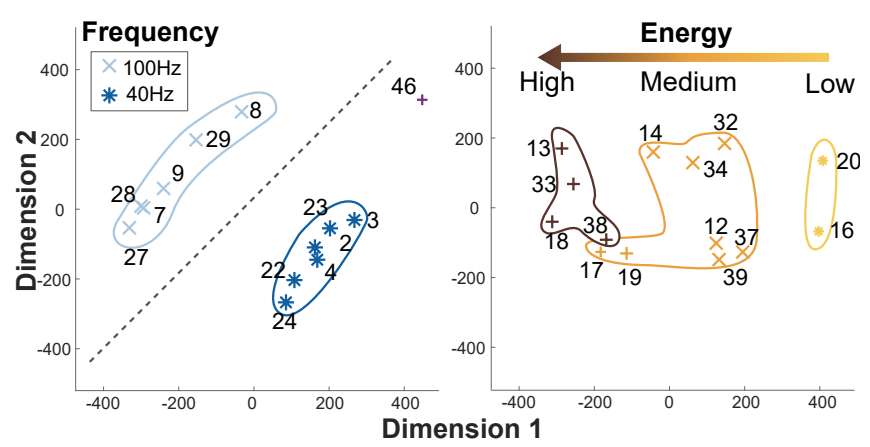

Figure 9. Sub-MDS plots for blue group (left) and yellow group (right). SS values are 0.150 and 0.165 , respectively.

$\mathrm{R} 50$, or R100). Therefore, using a low frequency and a relatively high-energy envelope enables vibrotactile augmentation to elicit a highly-stiff and rubbery sensation.

The blue group appears to be more scattered than the other groups, so we applied sub-MDS to the dissimilarity data of only this group. Results are shown in Figure 9, clarifying that the buttons can be categorized further into 40 and $100 \mathrm{~Hz}$. The physical button \#46 is rather isolated from the augmented buttons, with similar distances from the two frequency groups. Hence, the specific sensations of the augmented buttons seem to have subtle but consistent differences.

The last group, yellow, has the rest of the augmented buttons: \#12, \#13, \#14, \#16, \#17, \#18, \#19, \#20, \#32, \#33, \#34, \#37, $\# 38$, and \#39, with no physical buttons. All of them use vibrations of high frequency $(250$ or $400 \mathrm{~Hz})$ and mediumor high-energy envelope (DS, R50, or R100), with only two exceptions of \#16 and \#20 that have low-energy envelopes. These augmented buttons of high frequency and relatively high-energy envelope seem to deliver unique feels that are quite distinguished from those of the seven physical buttons. The sub-MDS results of this group are also shown in Figure 9, where the envelope energy is highly correlated with the perceptual dissimilarity.

\section{Augmentation Effects of Design Variables}

We can summarize the effects of the vibration design variables in modulating the feels of augmented physical buttons. For further support, the centroids are shown for each design variable in Figure 10. In the perceptual space, changing vibration frequency from 40 to $400 \mathrm{~Hz}$ moves the centroid from the right end to the left end. Envelope has a similar critical effect by translating the centroid vertically. In contrast, envelope shape (rectangular or decaying) and amplitude are much less important. Their centroids are gathered in a small area. Signal duration is correlated to envelope in our design, and its effects are not examined independently. All these observations are in good agreement with the clustering results of the buttons.

The criteria for similarity judgment mentioned by the majority of the participants were the frequency, (perceptual) strength, and duration of a button click sensation. These are consistent with our analysis results made using the perceptual space and the button groups. However, the fact that vibration amplitude has a minor role in similarity judgment is against our initial ex-

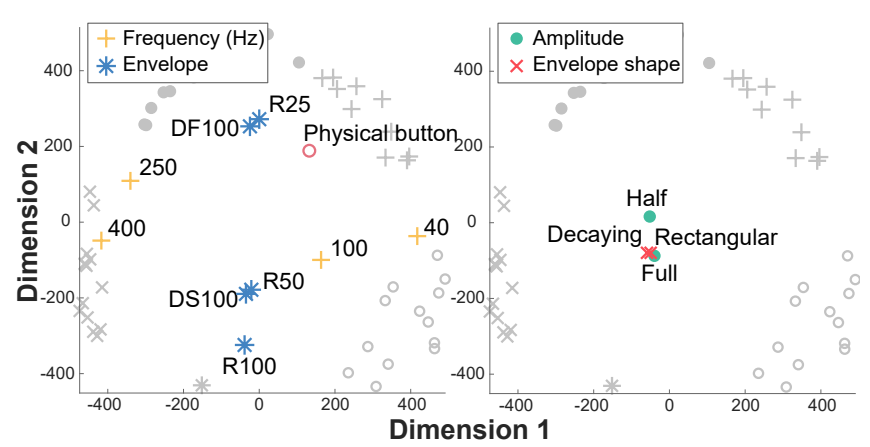

Figure 10. Centroids for four design variables. Frequency and envelope (left) and envelope shape and amplitude (right).

pectation. It seems that for the judgment of perceptual strength, the participants relied more on the combination of envelope shape and duration and rather ignored the pure amplitude level of vibration. An analogy can be that the perceptual strength of music is not related to sound intensity when we say that rock music sounds more powerful (in its nature) than classical music.

Our reports in this section are based mostly on the perceptual similarity between augmented and physical buttons. We further impose external validity in the next section by associating the perceptual space with linguistically meaningful axes.

\section{EXPERIMENT 2: PERCEPTUAL DIMENSIONS}

The next experiment was to describe the perceptual attributes of the buttons using adjective pairs and semantic words by projecting an external coordinate frame into the perceptual space found in Experiment 1. Participants' task was to rate the subjective feel of each button reflected in the words.

\section{Methods}

\section{Participants}

The 20 participants of Experiment 1 also participated in this experiment. They had a sufficient rest for a few ten minutes to prevent fatigue and tactile adaptation between Experiment 1 and 2 . They received approximately 20 USD as compensation.

\section{Apparatus and Experimental Conditions}

The experimental apparatus and conditions were the same as in Experiment 1.

\section{Procedure}

The task was an adjective rating using seven bipolar adjectives with opposing meanings and three semantic words, as listed in Table 2. The adjectives were chosen from the literature on haptic texture perception and articles about switches and buttons [17, 25, 36, 38].

\begin{tabular}{ccc|cc}
\hline$\#$ & Adjective 1 & Adjective 2 & $\#$ & Semantic \\
\hline 1 & Sharp & Blunt & 8 & Rubbery \\
2 & Even & Bumpy & 9 & Spongy \\
3 & Light & Heavy & 10 & Metallic \\
4 & Smooth & Sticky & & \\
5 & Soft & Hard & & \\
6 & Shallow & Deep & & \\
7 & Cheap & Luxurious & & \\
\hline
\end{tabular}

Table 2. Adjective pairs and semantic words used in Experiment 2. 

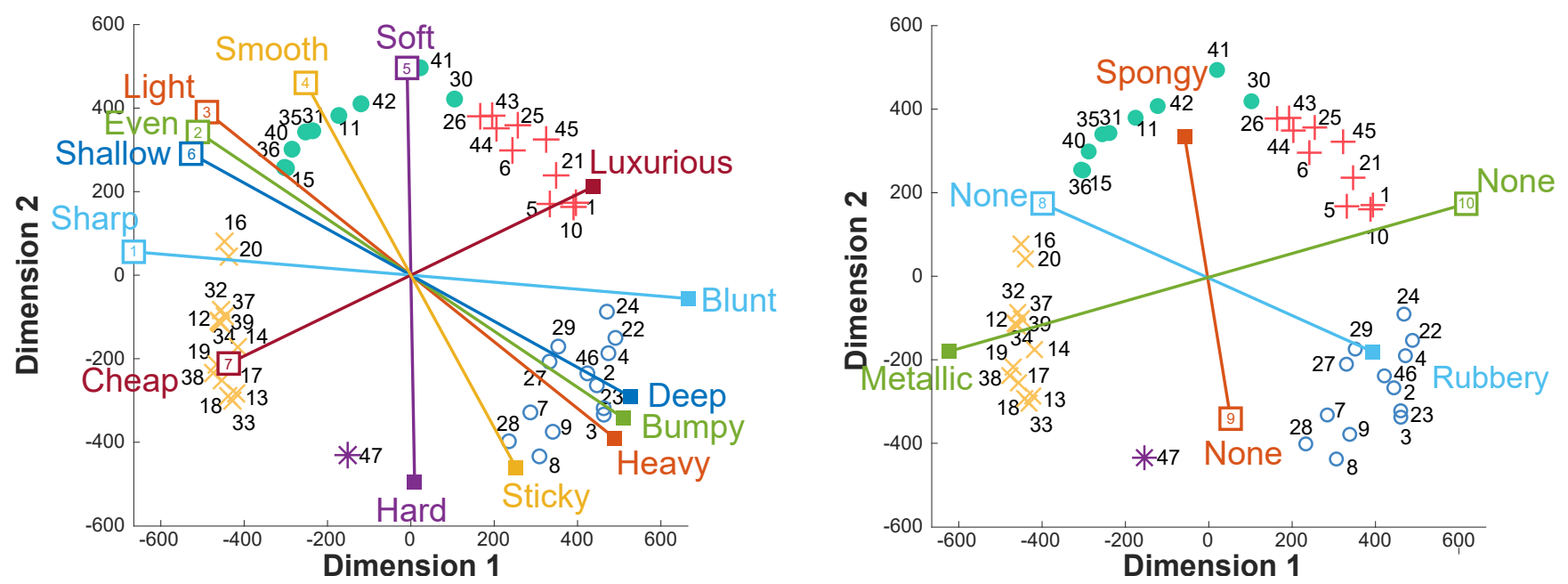

Figure 11. Adjective pairs regressed into the 2D perceptual space (left), and semantic words regressed into the space (right). The length of each axis is proportional to the $R^{2}$ value of the regression, indicating the degree of fit.

In each trial, participants perceived the stimulus by pressing the designated button on a GUI as many times as they wanted. Then they entered scores for the ten adjectives using ten sliders (127 $\mathrm{mm}$ long on the screen, following the recommendation of [52]). Each slider was labeled with the corresponding words in both our native language and English at adequate positions. All sliders were centered at the beginning of each trial. The 47 augmented and physical buttons were presented in random order to each participant. Participants wrote down their evaluation criteria at the end of the experiment. The experiment took $50 \mathrm{~min}$ on average.

\section{Data Analysis}

The bipolar scores were linearly converted to 0 for adjective 1 and 100 for adjective 2. The unipolar scores were rated from 0 to 100 . The data was averaged across the participants. For each adjective pair/semantic word, we applied multiple linear regression with the button coordinates recovered from the perceptual space as independent variables and the subjective score as a dependent variable. Then we projected each adjective pair/semantic word to a line in the perceptual space, which passes the origin and has a direction proportional to the standardized regression coefficients $[16,38]$. Each projected line represents a perceptual axis along which the feeling represented by the adjective pair/semantic word varies. A button position in the perceptual space is projected to a perceptual axis to recover the extent of the button's sensation described by the adjective pair/semantic word [17]. In this way, each button is assigned with numerical coordinates associated with a few adjectives, like colors represented in a color space.

\section{Results}

Figure 11 shows the main results of Experiment 2: the perceptual axes in the 2D perceptual space for the seven adjective pairs and three semantic words. The data plots and detailed statistics of multiple linear regression are available in the supplemental material for all adjective pairs and semantic words.

Since the perceptual space is $2 \mathrm{D}$, finding only two primary perceptual dimensions is sufficient to represent all the buttons

\begin{tabular}{lccccccc}
\hline & 1 & 2 & 3 & 4 & 5 & 6 & 7 \\
\hline 1 (Sharp-Blunt) & 0 & 27.3 & 34.1 & 57.0 & $\mathbf{8 4 . 3}$ & 22.3 & 30.7 \\
2 (Even-Bumpy) & & 0 & 6.8 & 29.8 & 57.0 & 5.0 & 58.0 \\
3 (Light-Heavy) & & & 0 & 22.9 & 50.2 & 11.8 & 64.8 \\
4 (Smooth-Sticky) & & & & 0 & 27.3 & 34.8 & $\mathbf{8 7 . 7}$ \\
5 (Soft-Hard) & & & & & 0 & 62.0 & 65.0 \\
6 (Shallow-Deep) & & & & & 0 & 53.0 \\
7 (Cheap-Luxurious) & & & & & & 0 \\
\hline
\end{tabular}

Table 3. Intersecting angles in degrees between adjectival axes.

\begin{tabular}{lccc}
\hline & 8 & 9 & 10 \\
\hline 8 (Rubbery) & 0 & 61.8 & 36.1 \\
9 (Spongy) & & 0 & $\mathbf{8 2 . 1}$ \\
10 (Metallic) & & & 0 \\
\hline
\end{tabular}

Table 4. Intersecting angles in degrees between semantic axes.

using coordinates. To this end, we first attend to $R^{2}$ of regression. According to Hollin et al. [16], qualitative scales with $R^{2} \geq 0.7$ are substantial, and $R^{2} \geq 0.5$ is regarded as moderately high for perceptual dimensions because of generally large subjective variability in perceptual studies. The $R^{2}$ values were $0.91,0.75,0.82,0.62,0.57,0.73,0.48,0.39$, 0.27 , and 0.85 , in the order of Table 2 . Thus, six adjective pairs of sharp-blunt, even-bumpy, light-heavy, smooth-sticky, soft-hard, and shallow-deep and one semantic word of metallic are adequate on the basis of $R^{2}$, and one adjective pair cheap-luxurious is at the borderline.

Second, mutually orthogonal axes are preferred as perceptual dimensions. The intersecting angles between the perceptual axes are shown in Table 3 and 4. The two pairs, (sharp-blunt, soft-hard) and (smooth-sticky, cheap-luxurious), are almost perpendicular with the intersecting angles of $84.3^{\circ}$ and $87.7^{\circ}$, respectively. In contrast, the even-bumpy, light-heavy, and shallow-deep axes nearly overlap each other with very small intersecting angles. Among the semantic axes, spongy and metallic have a large intersecting angle $\left(82.1^{\circ}\right)$.

Consequently, we recommend sharp-blunt and soft-hard as the two main perceptual dimensions to account for the perception of augmented and physical buttons. sharp-blunt has a very 
high linear correlation with the data $\left(R^{2}=0.91\right)$. soft-hard is nearly orthogonal $\left(84.3^{\circ}\right)$ to sharp-blunt with a good degree of fit $\left(R^{2}=0.57\right)$. The button are well clustered into the four quadrants partitioned by the sharp-blunt and soft-hard axes (Figure 11). Their interpretations of button-click sensations are also direct and intuitive.

For semantic words, metallic is the best perceptual dimension $\left(R^{2}=0.85\right)$. It is paired better with spongy with the intersecting angle of $82.1^{\circ}$ than rubbery, but both semantic words have low $R^{2}$ values $(<0.4)$. Thus, we should suggest metallic and spongy as the best two perceptual dimensions using semantic words, but the confidence on spongy is relatively low.

\section{Discussion}

\section{Primary Perceptual Dimensions}

The experimental results elucidate that sharp-blunt is the most appropriate perceptual dimension for represent the sensations of operating the physical and vibration-augmented buttons. This dimension is highly correlated with vibration frequency. In Figure 11, the high-frequency augmented buttons (green and yellow groups) are projected onto the sharp region of the dimension, whereas the low-frequency buttons (red and blue groups) are to the blunt region. The other primary perceptual dimension, soft-hard, is associated with the energy of vibration envelope. The augmented buttons with low-energy envelope (green and red groups) are projected to the soft side of the dimension, and those with medium- or high-energy envelope (yellow and blue groups) are to the hard side. These behaviors are consistent with the distribution of the centroids by frequency and envelope in Figure 10. Hence, the perceptual properties of the four button groups can be labeled as the green group: (sharp, soft), the red group: (blunt, soft), the blue group (blunt, hard), and the yellow group: (sharp, hard).

The percept of each button can be represented by its coordinates $(b, h)$, where $b$ is its position projected to the sharp-blunt axis and $h$ is that to the soft-hard axis. For example, the base physical button \#41 has $(b, h)=(-14,-496)$, while the augmented button \#23 in the blue group has $(b, h)=(485,331)$; see the supplemental material for the complete list. This metric nature may greatly facilitate understanding the perceptual relations between various vibration-augmented buttons, as does the HSV model for color perception and representation.

\section{Alternative Perceptual Dimensions}

Depending on applications, other perceptual dimensions can be more appropriate to the context. For example, for the use of augmented buttons in an expensive car, a metaphorical dimension of cheap-luxurious $\left(R^{2}=0.48\right)$ can be valuable in our adjective set. Such context-adapted use is applicable to all the perceptual dimensions shown in Figure 11.

Whether sharp-blunt and soft-hard are the best perceptual dimensions for other types of buttons, e.g., only physical buttons or virtual buttons, requires further verification. For instance, Chen et al. concluded that shallow-deep is the dominant perceptual axis to represent the manual key-clicks of various physical buttons that have considerable travel distances [7].

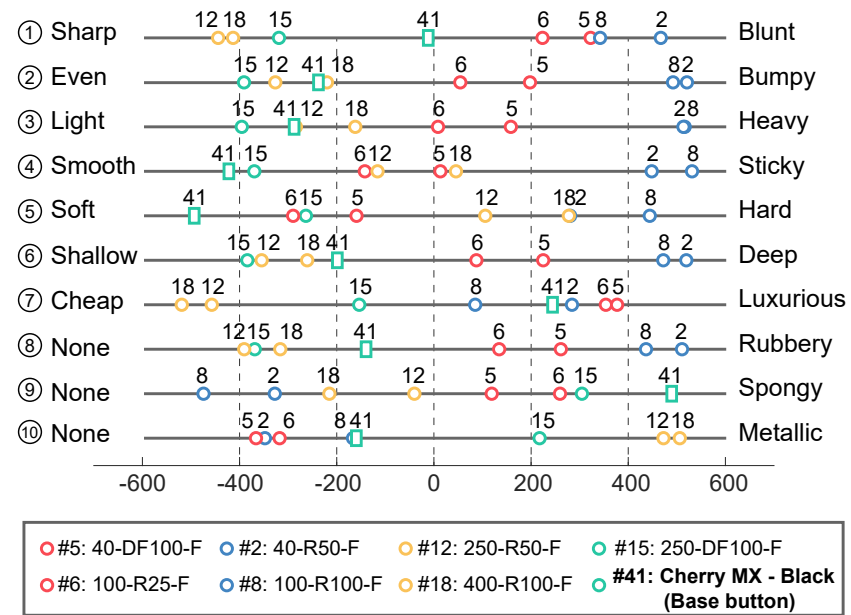

Figure 12. Projection of representative augmented buttons to adjectival and semantic axes. The base button \#41 has a box shape. Points are color-coded according to their groups.

\section{Designing Augmented Buttons of Specific Properties}

Here we discuss how to determine the values of the design variables in order to make a vibration-augmented button that has specific perceptual properties. For illustration, each button group is further split into two subgroups, and one button near the centroid of each subgroup is chosen as a representative. These buttons are \#15 and \#41 (green), \#5 and \#6 (red), \#2 and \#8 (blue), and \#12 and \#18 (yellow). They are projected to the seven adjectival and three semantic axes. Their coordinates are shown in Figure 12, where the axes are also indicated by circled numbers for simplicity. Let $p(\# j$, i $)$ be the projected coordinate of button $\# j$ to axis $i$.

As for the two primary perceptual dimensions:

sharp-blunt (compare the button coordinates marked on axis 1 in Figure 12) For the base physical button \#41, $p(\# 41,1)=-14$ (close to the neutral position 0). Adding a high-frequency vibration (\#12,\#15, and \#18; all 250 or $400 \mathrm{~Hz}$; see the legend at the bottom of Figure 12) makes its feel sharper, but adding a low-frequency vibration (\#2, \#5, \#6, and \#8; all 40 or $100 \mathrm{~Hz}$ ) makes it blunter.

soft-hard (axis 5 ) $p(\# 41,5)=-496$, corresponding to feeling extremely soft. Augmenting the base button with any vibration increases the hardness. The extent of hardness increase is positively correlated with the vibration envelope energy, as $p(\# 6,5)<p(\# 15,5)<p(\# 5,5)<$ $p(\# 12,5)<p(\# 18,5)=p(\# 2,5)<p(\# 8,5)$, and the envelopes of the seven buttons are R25, DF100, DF100, $R 50, R 100, R 50$, and R100, respectively; see (3) for the energy relations among the envelopes.

Similarly, for the rest of the adjectival and semantic axes:

even-bumpy (axis 2 ) $p(\# 41,2)=-240$, indicating that the base button feels fairly even. Similar to sharp-blunt, evenness increases if the base button is augmented with a high-frequency vibration (\#12, \#15, and \#18), while bumpiness increases if augmented with a low-frequency vibration (\#2, \#5, \#6, and \#8). 
light-heavy (axis 3 ) $p(\# 41,3)=-290$. The base button is perceived to be quite light, and it can be made even lighter by augmentation with a high-frequency, low-energy envelope vibration \#15: 250-DF-F. Augmentation with other low-frequency or higher-energy envelope vibrations increases the heaviness of sensation.

smooth-sticky (axis 4 ) $p(\# 41,4)=-425$. The base button feels the smoothest, and any vibrotactile augmentation makes the sensation sticker, similar to soft-hard.

shallow-deep (axis 6 ) $p(\# 41,6)=-201$. The base button has a moderate shallow sensation. High-frequency augmentation leads to an increase in shallowness (\#12, \#15, and $\# 18$ ), while low-frequency augmentation makes the sensation deeper (\#2, \#5, \#6, and \#8).

cheap-luxurious (axis 7$) p(\# 41,7)=242$, so the base physical button feels fairly luxurious. It is made more luxurious by vibrotactile augmentation with a low-frequency and low-energy envelope (\#2, \#5, and \#6). Using a high frequency or high-energy envelope induces the augmented button to feel cheaper (\#8, \#12, \#15, and \#18).

rubbery (axis 8 ) $p(\# 41,8)=-142$. The base button does not feel like a rubber (note the sign in Figure 12). Lowfrequency augmentation (\#2, \#5, \#6, and \#8) amplifies the rubber-like sensation, while high-frequency augmentation (\#12,\#15, and \#18) reduces it.

spongy (axis 9 ) $p(\# 41,9)=487$, and the base button feels the spongiest. Any augmentation makes it less spongy.

metallic (axis 10) $p(\# 41,10)=-166$. The base button is not metallic. Adding a low-frequency vibration (\#2, \#5, \#6, and \#8) makes it feel even less metallic. High-frequency augmentation renders it to be much more metallic (\#12, \#15, and \#18) by a greater extent.

Recall that in the discussion above, the augmentation effects on rubbery and spongy must be applied with care because of their low $R^{2}$ values.

\section{POTENTIAL USE SCENARIOS}

As substantiated by the two perceptual experiments, vibrationaugmented physical buttons provide unique and diverse haptic sensations despite the simplicity of hardware design and operation. In principle, the programmable vibrotactile feedback indicates additional information while the user presses a button, that is, enters an input command associated with the button. Therefore, the feedback can be about the state or result of interaction. This new mode of information display can be instrumental in many interaction scenarios.

As for state feedback, there can be many examples. The augmented feedback can improve entertainment applications to deliver more immersive and engaging user experiences. When playing a game, the navigation keys on the gamepad can be made to feel bumpy (button \#23: 40-R100-H; see Figure 11) when the game character walks on uneven ground, or sticky (button \#8: 100-R100-F) on muddy ground. Tactile feedback for the firing keys can be altered depending on the weapon (pistol, shotgun, cannon, etc.). We can also express context-dependent buttons. In a soccer game, there can be a button that leads to different actions during attack or defense. Providing different click sensations may enable the user to stay aware of the current context with a very light mental workload, or even unconsciously after prolonged use.

For electronic devices, consider an office telephone, which notifies the presence of the missed calls or messages stored in the cue. This is typically done by displaying a visual sign. As users do not always notice the visual signal, we can issue highly distinctive augmented feedback when they press any keys to use the phone. The feedback may contain more information. For instance, the feedback can be very soft, smooth, and light using button \#31: 250-R25-H for calls from a beloved someone. Button \#3: 40-R100-F with a heavy and bumpy sensation may be more adequate for a call from the boss at work. Here the button feedback works as a tactile icon $[6,37]$, conveying the abstract system state via touch.

Touch is a very effective modality for affective interfaces, and a car that has physical buttons with programmable feel is imaginable. A driver may choose the buttons' feel from presets as s/he does for the visual theme on the information display. This way, the car's haptic experiences can be customized to the driver: the system state that the augmented feedback represents.

Moving one step further, vibrotactile augmented feedback can be associated with the immediate system response to the user's key input. Normally, the augmented feedback is turned off, and the user perceives the original feedback of the button implying that the input command has been or will be executed without a problem. However, if the command is processed immediately and something abnormal, e.g., an error, occurs before the key press is completed, a vibration signal can be quickly superimposed to the button's restoration response- the average keystroke duration is around $80 \mathrm{~ms}$ [49], and modern computers are extremely fast. Perhaps we should do so using a sharp and hard augmented button like \#18: 400-R100-F. This approach, if implemented adequately to the buttons in a keyboard and a mouse, may be able to complement the usual error sounds, or become an alternative.

All of the above improvements require simple vibrotactile actuators to be added to plain physical buttons.

\section{DESIGN GUIDELINES}

From our results, major design guidelines of effective vibration augmentation for physical buttons are drawn as follows:

1. For the base physical button that has a linear response (no clear buckling) with a decent displacement range (4 mm), haptic augmentation using vibrotactile feedback is a very effective technique in expanding button click sensations.

2. The haptic percepts of vibration-augmented buttons are well expressed in a 2D perceptual space with the two perceptual dimensions of sharp-blunt and soft-hard.

3. Vibration frequency is a critical design variable for the feel of an augmented button. Using a high frequency makes the button feel shaper, and a low frequency blunter. 
4. The energy of a vibration determined by envelope shape and duration is another primary design variable. Augmentation with a high-energy envelope increases the hardness in perception, while a low-energy envelope the softness.

5. Envelope shape (rectangular or decaying) and vibration amplitude (full or half) have little effects on haptic sensation augmentation.

6. Our vibrotactile augmentation method systematically influences the other adjective pairs of even-bumpy, light-heavy, smooth-sticky, shallow-deep, and cheap-luxurious. For details, see the section Designing Augmented Buttons of Specific Properties, where we explain how to determine the values of the design variables to vary the sensations along the adjectival axes.

7. Strong augmentation with a high- and low-frequency vibration increases the sensations of metallic and rubbery, respectively.

8. Weak augmentation of the base linear physical button with a low-frequency vibration renders a similar sensation to that of a real physical button that exhibits a mild buckling effect.

\section{CONCLUSIONS}

This paper has explored a design space of a haptic augmentation technique that enables modulation of the feel of a real physical button by providing vibrotactile feedback. For a demonstration of the concept, we designed a large number of augmented buttons for one base physical button by varying the frequency, amplitude, envelope, and duration of vibration. Two user experiments were conducted, and their results offer the 2D perceptual space and two primary perceptual dimensions that quantify the perceptual effects of vibrotactile button augmentation in a metric space. Design guidelines for effective button augmentation are also summarized.

To our knowledge, this was the first study to elucidate the perceptual effects of physical button augmentation using vibration. As such, we are left with many important research questions. For example, the vibration initiation time was fixed in this study, but what would happen if we control it using an additional sensor? The base physical button used was a linear switch, but can we elicit pronounced augmentation effects from the physical switches that have strong buckling responses? Moreover, as vibrotactile feedback can serve as an ambient interface for many virtual controls [48], we can extend the augmentation technique to other physical controls, e.g, expression of various textures by adding vibration signals to a physical slider [55]. Haptic augmentation of other physical controls has a vast space to delve into.

\section{ACKNOWLEDGMENTS}

This work was supported by Institute for Information \& communications Technology Promotion (IITP) grants funded by the Korea government (MSIP) (No.2017-0-00179, HD Haptic Technology for Hyper Reality Contents) \& (No.2020-0-00594, Morphable Haptic Controller for Manipulating VR · AR Contents).

\section{REFERENCES}

[1] Jason Alexander, John Hardy, and Stephen Wattam. 2014. Characterising the Physicality of Everyday Buttons. In Proceedings of the ACM International Conference on Interactive Tabletops and Surfaces (ITS). ACM, 205-208. D0I :

http://dx.doi.org/10.1145/2669485.2669519

[2] Apple. 2020. Haptics - User Interaction - iOS - Human Interface Guidelines - Apple Developer. (2020).

Retrieved April 30, 2020 from https://developer.apple. com/design/human-interface-guidelines/ios/ user-interaction/haptics/.

[3] Olivier Bau and Ivan Poupyrev. 2012. REVEL: Tactile Feedback Technology for Augmented Reality. ACM Transactions on Graphics 31, 4 (2012), 1-11. DOI : http://dx.doi.org/10.1145/2185520.2185585

[4] Stanley J. Bolanowski, George A. Gescheider, Ronald T. Verrillo, and Christin M. Checkosky. 1988. Four Channels Mediate the Mechanical Aspects of Touch. Journal of the Acoustical Society of America 84, 5 (1988), 1680-1694. DOI : http://dx.doi.org/10.1121/1.397184

[5] Stephen Brewster, Stephen Brewster, Faraz Chohan, and Lorna Brown. 2007. Tactile Feedback for Mobile Interactions. In Proceedings of the SIGCHI conference on Human factors in Computing Systems (CHI). ACM, 159-162. DOI :

http://dx.doi.org/10.1145/1240624.1240649

[6] Stephen Brewster and Lorna M Brown. 2004. Tactons: Structured Vibrotactile Messages for Non-Visual Information Display. In Proceedings of the fifth conference on Australasian user interface (AUIC). Australian Computer Society, 15-23. DOI : http://dx.doi.org/10.5555/976310.976313

[7] Hsiang Yu Chen, Jaeyoung Park, Hong Z. Tan, and Steve Dai. 2011. Design and Evaluation of Identifiable Key-Click Signals for Mobile Devices. IEEE Transactions on Haptics 4, 4 (2011), 229-241. DOI : http://dx.doi.org/10.1109/TOH.2011.21

[8] CHERRY Corperation. 2020. MX Series Keyswitches. (2020). Retrieved April 30, 2020 from https://www . cherrymx.de/en/mx-original/mx-black.html.

[9] Heather Culbertson and Katherine J. Kuchenbecker. 2017. Ungrounded Haptic Augmented Reality System for Displaying Roughness and Friction. IEEE/ASME Transactions on Mechatronics 22, 4 (2017), 1839-1849. DOI : http://dx.doi.org/10.1109/TMECH.2017.2700467

[10] Stephanie Dabic, Jordan Navarro, Jean Marc Tissot, and Remy Versace. 2013. User Perceptions and Evaluations of Short Vibrotactile Feedback. Journal of Cognitive Psychology 25, 3 (2013), 299-308. DOI: http://dx.doi.org/10.1080/20445911.2013.768997

[11] Masaaki Fukumoto and Toshiaki Sugimura. 2001. Active Click: Tactile Feedback for Touch Panels. In 
Proceedings of the SIGCHI conference on Human factors in Computing Systems (CHI). ACM, 121-122. DOI : http://dx.doi .org/10.1145/634067.634141

[12] George A Gescheider. 1975. Psychophysics: the Fundamentals. Psychology Press.

[13] Taku Hachisu and Hiroyuki Kajimoto. 2013. HACHIStack: Dual-Layer Photo Touch Sensing for Haptic and Auditory Tapping Interaction. In Proceedings of the SIGCHI conference on Human factors in Computing Systems (CHI). ACM, 1411. DOI : http://dx.doi.org/10.1145/2470654.2466187

[14] Taku Hachisu and Hiroyuki Kajimoto. 2017. Vibration Feedback Latency Affects Material Perception during Rod Tapping Interactions. IEEE Transactions on Haptics 10, 2 (2017), 288-295. DOI : http://dx.doi.org/10.1109/ТОН.2016.2628900

[15] Eve Hoggan, Stephen A Brewster, and Jody Johnston. 2008. Investigating the Effectiveness of Tactile Feedback for Mobile Touchscreens. In Proceedings of the SIGCHI conference on Human factors in Computing Systems (CHI). ACM, 1573-1582. DOI : http://dx.doi.org/10.1145/1357054.1357300

[16] Mark Hollins, Richard Faldowski, Suman Rao, and Forrest Young. 1993. Perceptual Dimensions of Tactile Surface Texture: A Multidimensional Scaling Analysis. Perception \& Psychophysics 54, 6 (1993), 697-705. DOI : http://dx.doi .org/10.3758/BF03211795

[17] Inwook Hwang and Seungmoon Choi. 2010. Perceptual Space and Adjective Rating of Sinusoidal Vibrations Perceived via Mobile Device. In Proceedings of IEEE Haptics Symposium (HAPTICS). IEEE, 1-8. DOI : http://dx.doi.org/10.1109/HAPTIC. 2010.5444692

[18] Inwook Hwang, Jeongil Seo, and Seungmoon Choi. 2017. Perceptual Space of Superimposed Dual-frequency Vibrations in the Hands. PloS one 12, 1 (2017), e0169570. DOI :

http://dx.doi.org/10.1371/journal . pone.0169570

[19] Inwook Hwang, Jongman Seo, Myongchan Kim, and Seungmoon Choi. 2013. Vibrotactile Perceived Intensity for Mobile Devices as a Function of Direction, Amplitude, and Frequency. IEEE Transactions on Haptics 6, 3 (2013), 352-362. DOI :

http://dx.doi.org/10.1109/TOH. 2013.2

[20] Seokhee Jeon and Seungmoon Choi. 2009. Haptic Augmented Reality: Taxonomy and an Example of Stiffness Modulation. Presence: Teleoperators and Virtual Environments 18, 5 (2009), 387-408. DOI : http://dx.doi.org/10.1162/pres.18.5.387

[21] Seokhee Jeon and Seungmoon Choi. 2011. Real Stiffness Augmentation for Haptic Augmented Reality. Presence: Teleoperators and Virtual Environments 20, 4 (2011), 337-370. DOI :

http://dx.doi.org/10.1162/PRES_a_00051
[22] Seokhee Jeon, Seungmoon Choi, and Matthias Harders. 2012. Rendering Virtual Tumors in Real Tissue Mock-Ups Using Haptic Augmented Reality. IEEE Transactions on Haptics 5, 1 (2012), 77-84. DOI : http://dx.doi.org/10.1109/ТОН.2011.40

[23] Seokhee Jeon, Seungmoon Choi, and Matthias Harders. 2015. Haptic Augmented Reality Taxonomy, Research Status, and Challenges. In Fundamentals of Wearable Computers and Augmented Reality. Vol. 16. CRC Press, Chapter 10, 31-35. DOI :

http://dx.doi.org/10.1162/pres.18.5.387

[24] Seokhee Jeon, Jean-Claude Metzger, Seungmoon Choi, and Matthias Harders. 2011. Extensions to Haptic Augmented Reality: Modulating Friction and Weight. In Proceedings of the IEEE World Haptics Conference (WHC). IEEE, 227-232. DOI : http://dx.doi.org/10.1109/WHC. 2011.5945490

[25] Anzu Kawazoe, Massimiliano Di Luca, and Yon Visell. 2019. Tactile Echoes: A Wearable System for Tactile Augmentation of Objects. In Proceedings of the IEEE World Haptics Conference (WHC). IEEE, 359-364. DOI : http://dx.doi.org/10.1109/WHC.2019.8816099

[26] Johan Kildal. 2010. 3D-Press: Haptic Illusion of Compliance when Pressing on a Rigid Surface. In Proceedings of International Conference on Multimodal Interfaces and the Workshop on Machine Learning for Multimodal Interaction, (ICMI-MLMI). ACM. DOI: http://dx.doi.org/10.1145/1891903.1891931

[27] Jin Ryong Kim and Hong Z Tan. 2014. A Study of Touch Typing Performance with Keyclick Feedback. In Proceedings of IEEE Haptics Symposium (HAPTICS). IEEE, 227-233. DOI : http://dx.doi .org/10.1109/HAPTICS.2014.6775459

[28] Sunjun Kim, Byungjoo Lee, and Antti Oulasvirta. 2018. Impact Activation Improves Rapid Button Pressing. In Proceedings of the SIGCHI conference on Human factors in Computing Systems (CHI). ACM, 1-8. DOI: http://dx.doi.org/10.1145/3173574.3174145

[29] Sunjun Kim and Geehyuk Lee. 2013. Haptic Feedback Design for a Virtual Button Along Force-Displacement Curves. In Proceedings of Annual ACM Symposium on User Interface Software and Technology (UIST). ACM, 91-96. DOI : http://dx.doi.org/10.1145/2501988.2502041

[30] Sunjun Kim, Jeongmin Son, Geehyuk Lee, Hwan Kim, and Woohun Lee. 2013. TapBoard: Making a Touch Screen Keyboard More Touchable. In Proceedings of the SIGCHI conference on Human factors in Computing Systems (CHI). ACM, 553. DOI: http://dx.doi.org/10.1145/2470654.2470733

[31] Katherine J. Kuchenbecker, Jonathan Fiene, and Günter Niemeyer. 2006. Improving Contact Realism through Event-Based Haptic Feedback. IEEE Transactions on Visualization and Computer Graphics 12, 2 (2006), 219-229. DOI : http://dx.doi .org/10.1109/TVCG.2006. 32 
[32] Kiuk Kyung, Jeongmook Lim, Yoan Lim, Suntak Park, Seungkoo Park, Inwook Hwang, Seungmoon Choi, Jongman Seo, Sangyoun Kim, Taeheon Yang, and Dongsoo Kwon. 2011. TAXEL: Initial Progress Toward Self-Morphing Visio-Haptic Interface. In Proceedings of the IEEE World Haptics Conference (WHC). IEEE, 37-42. DOI :

http://dx.doi.org/10.1109/WHC. 2011.5945458

[33] Jaebong Lee and Seungmoon Choi. 2013. Real-Time Perception-Level Translation from Audio Signals to Vibrotactile Effects. In Proceedings of the SIGCHI conference on Human factors in Computing Systems (CHI). ACM, 2567-2576. DOI :

http://dx.doi.org/10.1145/2470654.2481354

[34] Yichi Liao, Sunjun Kim, and Antti Oulasvirta. 2018. One Button to Rule Them All: Rendering Arbitrary Force-Displacement Curves. In Proceedings of Annual ACM Symposium on User Interface Software and Technology (UIST). ACM, 111-113. DOI : http://dx.doi.org/10.1145/3266037.3266118

[35] Yi-Chi Liao, Sunjun Kim, Byungjoo Lee, and Antti Oulasvirta. 2020. Button Simulation and Design via FDVV Models. In Proceedings of the SIGCHI conference on Human factors in Computing Systems (CHI). ACM. DOI : http://dx.doi.org/10.1145/3313831.3376262

[36] Quan Liu, Hong Z. Tan, Liang Jiang, and Yulei Zhang. 2018. Perceptual Dimensionality of Manual Key Clicks. In Proceedings of IEEE Haptics Symposium (HAPTICS), Vol. 2018-March. IEEE, 112-118. DOI : http://dx.doi.org/10.1109/HAPTICS.2018.8357162

[37] Karon Maclean and Mario Enriquez. 2003. Perceptual Design of Haptic Icons. In Proceedings of EuroHaptics. 351-363. DOI : http://dx. doi .org/10.3758/BF03194057

[38] Sunung Mun, Hojin Lee, and Seungmoon Choi. 2019. Perceptual Space of Regular Homogeneous Haptic Textures Rendered Using Electrovibration. In Proceedings of the IEEE World Haptics Conference (WHC). IEEE, Tokyo, Japan, 3-8. DOI : http://dx.doi.org/10.1109/WHC. 2019.8816143

[39] Daichi Ogawa, Taku Hachisu, and Hiroyuki Kajimoto. 2015. Multiple Texture Button by Adding Haptic Vibration to the Physical Button. In ACM SIGGRAPH Asia 2015 Haptic Media And Contents Design. ACM, 12. DOI:

http://dx.doi.org/10.1299/jsmermd.2015._2a1-b01_1

[40] Shogo Okamoto, Hikaru Nagano, and Yoji Yamada. 2012. Psychophysical Dimensions of Tactile Perception of Textures. IEEE Transactions on Haptics 6, 1 (2012), 81-93. DOI : http://dx . doi . org/10.1109/TOH. 2012 . 32

[41] Allison M. Okamura, Mark R. Cutkosky, and Jack Tigh Dennerlein. 2001. Reality-Based Models for Vibration Feedback in Virtual Environments. IEEE/ASME Transactions on Mechatronics 6, 3 (2001), 245-252. DOI : http://dx. doi .org/10.1109/3516.951362
[42] Chaeyong Park, Jaeyoung Park, Seungjae Oh, and Seungmoon Choi. 2019. Realistic Haptic Rendering of Collision Effects Using Multimodal Vibrotactile and Impact Feedback. In Proceedings of the IEEE World Haptics Conference (WHC). IEEE, Tokyo, 449-454. DOI : http://dx.doi .org/10.1109/WHC.2019.8816116

[43] Gunhyuk Park, Hojun Cha, and Seungmoon Choi. 2019. Haptic Enchanters: Attachable and Detachable Vibrotactile Modules and Their Advantages. IEEE Transactions on Haptics 12, 1 (2019), 43-55. DOI : http://dx.doi.org/10.1109/TOH.2018.2859955

[44] Gunhyuk Park and Seungmoon Choi. 2011. Perceptual Space of Amplitude-Modulated Vibrotactile Stimuli. In Proceedings of the IEEE World Haptics Conference (WHC). IEEE, 59-64. DOI :

http://dx.doi.org/10.1109/WHC. 2011.5945462

[45] Gunhyuk Park and Seungmoon Choi. 2017. A Physics-Based Vibrotactile Feedback Library for Collision Events. IEEE Transactions on Haptics 10, 3 (2017), 325-337. DOI : http://dx.doi .org/10.1109/ТOH.2016.2614804

[46] Gunhyuk Park, Seungmoon Choi, Kyunghun Hwang, Sunwook Kim, Jaecheon Sa, and Moonchae Joung. 2011. Tactile Effect Design and Evaluation for Virtual Buttons on a Mobile Device Touchscreen. In Proceedings of the International Conference on Human Computer Interaction with Mobile Devices and Services (MobileHCI). ACM, 11. DOI : http://dx.doi.org/10.1145/2037373.2037376

[47] Jerome Pasquero, Joseph Luk, Shannon Little, and Karon MacLean. 2006. Perceptual Analysis of Haptic Icons: An Investigation into the Validity of Cluster Sorted MDS. In Proceedings of Symposium on Haptic Interfaces for Virtual Environment and Teleoperator Systems (VR). IEEE, 437-444. DOI :

http://dx. doi .org/10.1109/HAPTIC.2006.1627122

[48] Ivan Poupyrev, Shigeaki Maruyama, and Jun Rekimoto. 2002. Ambient Touch: Designing Tactile Interfaces for Handheld Devices. In Proceedings of Annual ACM Symposium on User Interface Software and Technology (UIST). ACM, 51-60. DOI : http://dx.doi.org/10.1145/571985.571993

[49] David Rempel, Jack Dennerlein, CD Mote Jr, and Thomas Armstrong. 1994. A Method of Measuring Fingertip Loading During Keyboard Use. Journal of Biomechanics 27, 8 (1994), 1101-1104. DOI : http://dx.doi .org/10.1016/0021-9290(94)90227-5

[50] Joseph M. Romano and Katherine J. Kuchenbecker. 2012. Creating Realistic Virtual Textures from Contact Acceleration Data. IEEE Transactions on Haptics 5, 2 (2012), 109-119. DOI : http://dx.doi.org/10.1109/ТОН.2011.38 
[51] Bushra Sadia, Senem Ezgi Emgin, T Metin Sezgin, and Cagatay Basdogan. 2020. Data-Driven Vibrotactile Rendering of Digital Buttons on Touchscreens. Journal of Human Computer Studies 135, September 2019 (2020), 102363. DOI :

http://dx.doi.org/10.1016/j.ijhcs.2019.09.005

[52] Susan S. Shiffman, M .Lance Reynolds, and Forrest W. Young. 1981. Handbook of Multidimensional Scaling. New York, NY, USA: Academic Press (1981).

[53] Youngbo Aram Shim and Geehyuk Lee. 2018. Demonstrating Gamepad with Programmable Haptic Texture Analog Buttons. In Proceedings of Annual ACM Symposium on User Interface Software and Technology (UIST). ACM, 194-196. DOI : http://dx.doi.org/10.1145/3266037.3271648

[54] Sunghwan Shin and Seungmoon Choi. 2018. Effects of Haptic Texture Rendering Modalities on Realism. In Proceedings of the ACM Symposium on Virtual Reality Software and Technology (VRST). ACM, 1-5. DOI : http://dx.doi.org/10.1145/3281505.3281520

[55] Paul Strohmeier and Kasper Hornbæk. 2017. Generating Haptic Textures with a Vibrotactile Actuator. In Proceedings of the SIGCHI conference on Human factors in Computing Systems (CHI). ACM, 4994-5005. DOI : http://dx.doi.org/10.1145/3025453.3025812

[56] Kaoru Tashiro, Yuta Shiokawa, Tomotake Aono, and Takashi Maeno. 2009. Realization of Button Click Feeling by Use of Ultrasonic Vibration and Force Feedback. In Proceedings of the IEEE World Haptics Conference (WHC). IEEE, 1-6. DOI : http://dx.doi.org/10.1109/WHC.2009.4810877

[57] David Ternes and Karon E Maclean. 2008. Designing Large Sets of Haptic Icons with Rhythm. In Proceedings of EuroHaptics. Springer, 199-208. DOI : http://dx.doi.org/10.1007/978-3-540-69057-3_24

[58] Wouter M Bergmann Tiest and Astrid ML Kappers. 2006. Analysis of Haptic Perception of Materials by Multidimensional Scaling and Physical Measurements of Roughness and Compressibility. Acta Psychologica
121, 1 (2006), 1-20. DOI :

http://dx.doi.org/10.1016/j . actpsy.2005.04.005

[59] Ronald T. Verrillo, Anthony J. Fraioli, and Robert L. Smith. 1969. Sensation Magnitude of Vibrotactile Stimuli. Perception \& Psychophysics 6, 6 (1969), 366-372. DOI: http://dx.doi.org/10.3758/BF03212793

[60] Jonathan D. Victor, Syed M. Rizvi, and Mary M. Conte. 2017. Two Representations of a High-Dimensional Perceptual Space. Vision Research 137 (2017), 1-23. DOI : http://dx.doi.org/10.1016/j.visres.2017.05.003

[61] Thomas E von Wiegand, David W Schloerb, and W L Sachtler. 1999. Virtual Workbench: Near-Field Virtual Environment System with Applications. Presence 8, 5 (1999), 492-519. DOI : http://dx.doi.org/10.1162/105474699566422

[62] Dangxiao Wang, Kouhei Ohnishi, and Weiliang Xu. 2020. Multimodal Haptic Display for Virtual Reality: A Survey. IEEE Transactions on Industrial Electronics 67, 1 (2020), 610-623. DOI : http://dx.doi.org/10.1109/TIE.2019.2920602

[63] David W. Weir, Michael Peshkin, J. Edward Colgate, Pietro Buttolo, James Rankin, and Matthew Johnston. 2004. The Haptic Profile: Capturing the Feel of Switches. In Proceedings of International Symposium on Haptic Interfaces for Virtual Environment and Teleoperator Systems (HAPTICS). IEEE, 186-193. DOI : http://dx. doi .org/10.1109/HAPTIC.2004.1287195

[64] Shunsuke Yoshimoto, Yoshihiro Kuroda, Yuki Uranishi, Masataka Imura, and Osamu Oshiro. 2014. Roughness Modulation of Real Materials Using Electrotactile Augmentation. In Proceedings of EuroHaptics, Vol. 8618. Springer, 10-17. DOI: http://dx.doi .org/10.1007/978-3-662-44193-0

[65] Qasim Zaidi, Jonathan Victor, Josh McDermott, Maria Geffen, Sliman Bensmaia, and Thomas A Cleland. 2013. Perceptual Spaces: Mathematical Structures to Neural Mechanisms. Journal of Neuroscience 33, 45 (2013), 17597-17602. DOI : http://dx.doi.org/10.1523/JNEUROSCI. 3343-13.2013 